\title{
Analysis of community properties and node properties to understand the structure of the bus transport network
}

\author{
Yeran Sun ${ }^{\mathrm{a}, *}$, Lucy Mburu ${ }^{\mathrm{a}}$, Shaohua Wang ${ }^{\mathrm{b}}$ \\ ${ }^{a}$ GIScience Research Group, Institute of Geography, Heidelberg University, Berliner Strasse 48, Heidelberg 69120, Germany \\ ${ }^{\mathrm{b}}$ Institute of Geographic Sciences and Natural Resources Research, Chinese Academy of Sciences, Datun Road 11A, Beijing 100101, China
}

\section{H I G H L I G H T S}

- We analyze the community structure of the bus network and identify the important nodes in the network.

- The intra-urban bus network has a multi-community structure.

- The geographic characteristics of communities somewhat reflect the socio-economic division in the city.

- The majority of the important nodes (hubs) are clustered in the city center, implying the majority of bus lines are likely to go through the city center.

\section{A R T I C L E I N F O}

\section{Article history:}

Received 13 May 2015

Received in revised form 22 November

2015

Available online 22 January 2016

\section{Keywords:}

Bus networks

Community structure

Node prominence

Geographic characteristics

Network topology

\begin{abstract}
A B S T R A C T
Akin to most infrastructures, intraurban bus networks are large and highly complex. Understanding the composition of such networks requires an intricate decomposition of the network into modules, taking into account the manner in which network links are distributed among the nodes. There exists for each set of highly interlinked nodes little connectivity with the next set of highly interlinked nodes. This inherent property of nodes makes community detection a popular approach for analyzing the structure of complex networks. In this study, we attempt to understand the structure of the intraurban bus network of Ireland's capital city, Dublin in a two-step approach. We first analyze the modular structure of the network by identifying potential communities. Secondly, we assess the prominence of each network node by examining the module-based topological properties of the nodes. Results of this empirical study reveal a clear pattern of independent communities, indicating thus, an implicit multi-community structure of the intraurban bus network. Examination of the geographic characteristics of the identified communities shows a degree of socio-economic divisions of the Dublin city. Furthermore, a large majority of the important nodes (vital transportation hubs) are located at the city center, implying that most of the bus lines in Dublin city tend to intersect the city's core.
\end{abstract}

(c) 2016 Elsevier B.V. All rights reserved.

\section{Introduction}

A clear knowledge of the structure and properties of public transportation networks is crucial for the urban planning and administration, policy enforcement, and disaster management [1]. The literature suggests that urban infrastructures, such as the bus, subway, railway, and aircraft networks have inherent small-world properties [2], and a complex topological

\footnotetext{
* Correspondence to: Room 101, Berliner Strasse 48, Heidelberg 69120, Germany. Tel.: +49 6221 54 5528; fax: +49 6221544529 .

E-mail address: yeran.sun@geog.uni-heidelberg.de (Y. Sun).
} 
structure [3-11]. Bus networks are the most popular kind of intraurban public transportation networks in many cities, and previous research has strived to understand the statistical mechanics of these networks [1,8]. Exploratory research has led to the discovery of three coordinated properties that characterize the bus networks as complex: (1) the degree distribution of nodes follows a power law or an exponential law, (2) the relationship between the degree of nodes and their relative influence is positive and linear, and (3) bus networks exhibit a high clustering coefficient, suggesting that such networks are 'small-worlds' [1,8,12-14]. Complex networks exhibit an unequal degree distribution, and this distribution forms the basis for dividing the network into smaller clusters. A "community" is, thus, detected from nodes with strong internal connections and weak connections between different clusters, and each community corresponds to a network component or sub-network [15]. This study, therefore, applies a community-based approach to analyze different properties of the network structure.

The purpose of this study is to investigate the structure of an intraurban bus network. Specifically, we assess the network community structure and subsequently analyze the roles of nodes in the bus network. The remainder of this paper is organized as follows. Section 2 introduces the approach to analyzing the network structure, while Section 3 presents the empirical study and the relevant results, and a discussion of the results. We conclude with recommendations for future research.

\section{Methodology}

This section advances the approach adopted for analyzing the bus network structure. First we introduce the strategies for partitioning the network into communities and assessing the geographic characteristics and topological structure of the primary communities. Afterwards, the section presents the application of module-based measures to describe the role of nodes in the network. The advanced approach will be applied in the subsequent sections to identify and analyze important bus network nodes.

\subsection{Community detection}

Modularity-based measures have been proposed for community detection [16-18]. Given a partition of a complex network into modules (sub-networks), the network modularity, $M$ quantifies the strength of the division as:

$$
M=\sum_{j \in \mathrm{J}}\left[\frac{l_{j}}{L}-\left(\frac{d_{j}}{2 L}\right)^{2}\right]
$$

where $L$ is the number of network links, $l_{j}$ is the number of links between nodes of module $j$ (i.e., the number of intramodule links within module $j$ ), $d_{j}$ is the sum of degrees of all nodes within module $j$, and $2 L$ is the sum of the degrees of all nodes in the network. J is the set of modules divided. This definition of modularity can be justified. An accurate partition of the network into modules is designed to maximize the links within modules and minimize the links between modules. Accordingly, a high value of $M$ corresponds to a solid network partition. However, if the sole intention is to minimize the number of between-module links (or, equivalently, to maximize the number of within-module links) the optimal partition will consist of a single module and no between-module links. Eq. (1) alleviates this problem by imposing the constraint that $M$ is zero if the nodes are randomly located across the modules, or if all nodes belong to the same cluster [16,17].

Several modularity-based community detection algorithms have been proposed for partitioning complex networks, including FastGreedy [19], Spinglass [20], Walktrap [21], and Infomap [18]. A recent study by Ref. [15] comparing the community detection algorithms illustrated that Infomap outperforms the other widely used algorithms in its efficiency. This discovery guides the choice of the Infomap algorithm for community detection in the current study. Infomap detects the inherent community structure by minimizing the expected description length of a random walker's trajectory [18]. The algorithm employs the probability flow of random walks along a network as a proxy for information flow in the real system, and thus, it decomposes the network into modules by compressing a description of the probability flow [18].

\subsection{Assessing the influence of network nodes}

Two indicators are popular for measuring a node's influence within the network. The within-module degree $z$-score measures the degree centrality within a module, and the participation coefficient ( $p$-value) measures the amount of variation in intermodular node connections [7]. The rationale for these indicators is that nodes which possess similar topological properties have high probability for playing similar roles. Within the framework of a complex network, the role of a certain node, $v_{i}$ is defined by its location with reference to other nodes belonging to its module, and also by how well it connects to the nodes of other modules [7].

The modules of a complex network are structurally distinct in their organization, and they range from completely centralized modules (in which a few central nodes are linked to all other nodes) to completely decentralized (all nodes are similarly connected). It follows naturally, therefore, that nodes with similar roles will possess a relatively similar withinmodule connectivity. On the one hand, the $z$-score quantifies the degree of connectivity of a certain node, $v_{i}$ to the other 
nodes in its module, and it is denoted as:

$$
z_{v_{i}}=\frac{e_{j_{v_{i}^{\prime}}}-\bar{e}_{j^{\prime}}}{\sigma_{e_{j^{\prime}}}}
$$

where $e_{j_{v_{i}}}$ denotes the number of edges (links) of a node, $v_{i}$ to all other nodes within its module, $j^{\prime}$, and $\bar{e}_{j^{\prime}}$ denotes the average of $e_{j_{v_{i}}}$ over the nodes of module $j^{\prime}$. $\sigma_{e_{j^{\prime}}}$ corresponds to the standard deviation of $e_{j_{v_{i}}}$ over the nodes of module $j^{\prime}$. On the other hand, the $p$-value quantifies the degree of connectivity of node to other nodes outside its own module. This measurement is necessary because roles (e.g., broker) often arise due to the cross-module connectivity of nodes. Thus, it is possible that between any pair of nodes within a module which have equal $z$-score and only one node is cross-connected to other modules, there will be a difference in the amount of influence exerted. The participation coefficient, $p_{v_{i}}$ of node $v_{i}$ is expressed as:

$$
p_{v_{i}}=1-\sum_{j \in \mathrm{J}}\left(\frac{e_{j_{v_{i}}}}{e_{v_{i}}}\right)^{2}
$$

where $e_{j_{v i}}$ corresponds to the number of links of node $v_{i}$ to other nodes within module $j$, and $e_{v i}$ is the total degree of this node, irrespective of which module node the links connect to. J is the set of modules divided. $p_{v_{i}} \approx 1$ if the links of this node are uniformly distributed between modules, and $p_{v_{i}} \approx 0$ if all of its links fall within its own module, $j$. Thus, we hypothesize based on the literature, that the role of a node is largely determined by the measures of within-module degree and participation coefficient above [22,23]. We hence apply these measures to define how nodes are positioned with reference to their own modules and their connectivity with nodes of other modules.

\section{Empirical analysis}

This section discusses the structure of the bus network used for the empirical analysis. After introducing the network, we apply the approach for detecting communities. We thereafter analyze the geographic characteristics and topologies of the main communities identified. Using this knowledge the final step in this section is to identify and analyze the important nodes in the network.

\subsection{Case study and data}

The case study used for this analysis is the bus network in Dublin city, the capital and largest city of Ireland. The River Liffey divides the city into two parts: the Northside and the Southside. The experimental data set is the Dublin bus line data set offered by Dublinked (http://dublinked.com/datastore). From this data set, 4618 bus stops connected by 13,046 bus line segments were extracted. The bus line segment corresponds to the link connecting two consecutive bus stops on a bus line (see Fig. 1). The bus stops and bus line segments represent the network nodes and links (edges) respectively. From this data set we constructed a complex intraurban bus network comprising 4618 nodes and 13,046 edges.

\subsection{Detection and analysis of communities in the bus network}

\subsubsection{Detection of communities}

The Infomap algorithm used for the community detection analysis was implemented using the R package igraph [24]. After a series of 40 partitioning iterations, the best division was acquired with a modularity value of 0.191 . The most accurate split resulted in 35 communities or modules, $\{\mathrm{c} 1, \ldots, \mathrm{c} 35\} \subset \mathrm{J}$. Table 1 lists selected communities with priority on the number of intra-module links, the number of nodes and the sum of degrees of the nodes. We illustrate the calculation of the network modularity on the basis of the identified communities:

$$
\begin{aligned}
M & =\sum_{j \in \mathrm{J}}\left[\frac{l_{j}}{L}-\left(\frac{d_{j}}{2 L}\right)^{2}\right]=\left[\frac{l_{c 1}}{L}-\left(\frac{d_{c 1}}{2 L}\right)^{2}\right]+\left[\frac{l_{c 2}}{L}-\left(\frac{d_{c 2}}{2 L}\right)^{2}\right]+\cdots+\left[\frac{l_{c 35}}{L}-\left(\frac{d_{c 35}}{2 L}\right)^{2}\right] \\
& =\left[\frac{11677}{13046}-\left(\frac{23384}{2 * 13046}\right)^{2}\right]+\left[\frac{335}{13046}-\left(\frac{678}{2 * 13046}\right)^{2}\right]+\cdots+\left[\frac{1}{13046}-\left(\frac{2}{2 * 13046}\right)^{2}\right] \\
& =0.191 .
\end{aligned}
$$

\subsubsection{Analysis of primary communities}

The focus now shifts to the main communities with reference to the five most prominent modules listed in Table 1 as $1-5$. These modules have the largest number of intra-module links and the largest number of nodes, and together they account 


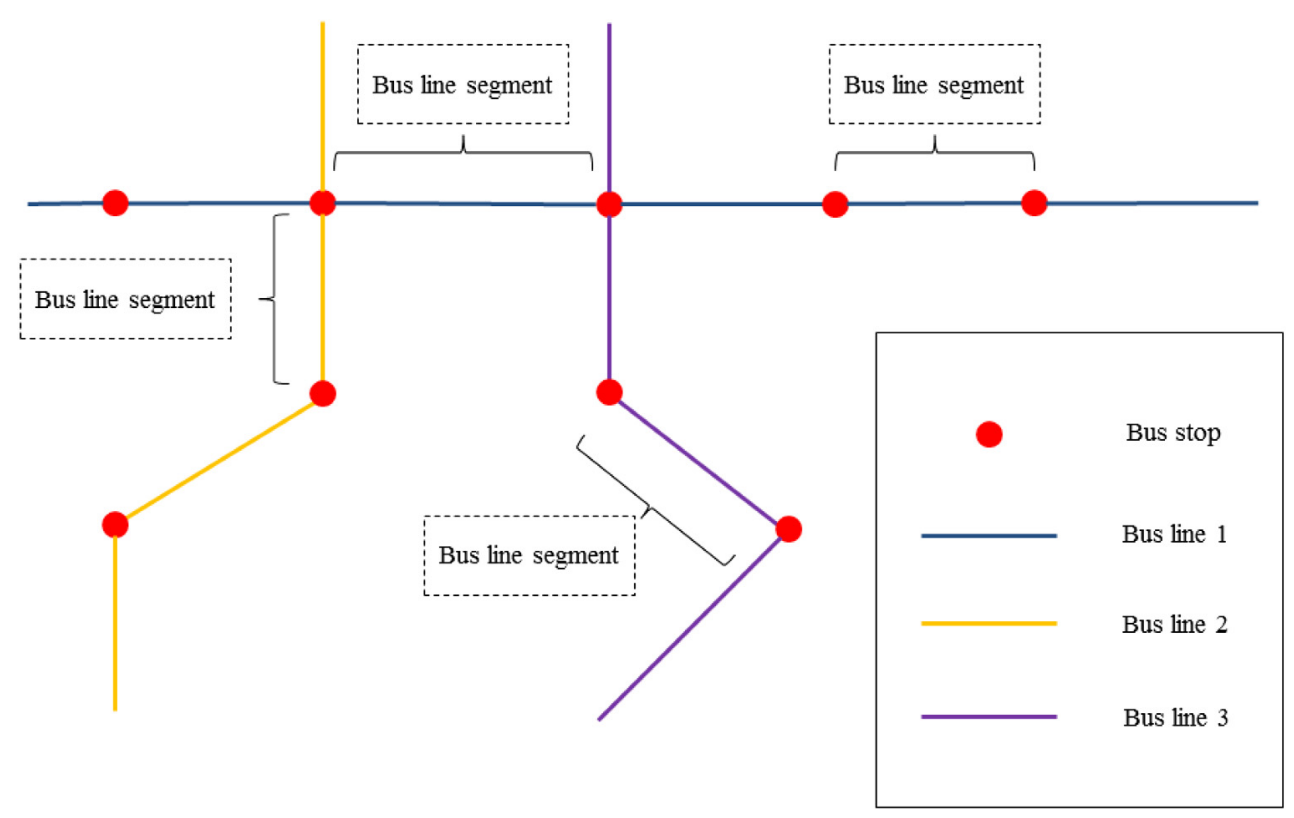

Fig. 1. Illustration of the bus line segment.

Table 1

Selected communities detected from the intraurban bus network, including intra-module based statistics (links count, nodes count, sum of node degrees).

\begin{tabular}{|c|c|c|c|}
\hline $\begin{array}{l}\text { Community } \\
\text { (module) }\end{array}$ & $\begin{array}{l}\text { Number of intra-module } \\
\text { (within-module) links }\end{array}$ & $\begin{array}{l}\text { Number of } \\
\text { within-module nodes }\end{array}$ & $\begin{array}{l}\text { Sum of node degrees of } \\
\text { within-module nodes }\end{array}$ \\
\hline c1 & 11,677 & 3474 & 23,384 \\
\hline c2 & 335 & 306 & 678 \\
\hline c3 & 148 & 98 & 305 \\
\hline c4 & 68 & 68 & 139 \\
\hline c5 & 47 & 47 & 95 \\
\hline$\ldots$ & & $\ldots$ & $\ldots$ \\
\hline c35 & 1 & 2 & 2 \\
\hline
\end{tabular}

for about $85 \%$ of all the network nodes. Specifically, the largest community (i.e., c1) contains 3474 nodes, thus accounting for about $75 \%$ of all nodes. In comparison to $\mathrm{c} 1$, the other communities, c2-c5 have a relatively insignificant number of intra-module links and nodes.

(1) Assessing the geographic characteristics of the primary communities

Fig. 2 maps the five largest communities. It can be noted that Community 4 has not been mapped, since this community falls outside the administrative boundaries of the Dublin city. Not surprisingly, however, the largest community (c1), covers almost the entire city. It is also interesting to observe that the second largest community (c2) covers the southern part of the city (traditionally called the Southside), whereas the other two communities, i.e., c3 and c5, cover mainly the northern part of the city (traditionally called the Northside). While the Northside and the Southside are not official administrative divisions of Dublin city, there is a socio-economic north-south division, with the River Liffey as the dividing line. This north-south division now presents a cultural and socio-economic stratification basis. For instance, the majority of the residents in Northside essentially comprise the working class, while residents of the Southside are likely to comprise the upper and middle class. A prominent discovery from the uncovered partitions is thus, that the geographic properties of the identified communities somewhat reflect the north-south sub-division of the Dublin city. Additionally, c2 looks like a L-shaped network that links the northern part with the western part of the city.

(2) Topologies of the main communities

A network's topology describes the arrangement of nodes and links within the network. Since a community corresponds to a sub-network, it is possible that different types of network topologies will characterize the different communities of a single network [25,26]. An overview of the basic network topologies (Fig. 3) allows us to account for the number of links and node degree (see Table 2). $n$ and $e$ represent the number of nodes and the number of links in the network respectively. $d$ represents node degree (the number of links of a node to other nodes).

In the line network, $d$ equals 1 for each of the external nodes, and $d$ equals 2 for all the other nodes. In the star network, all nodes are connected to the central node (hub). Thus, $e$ equals $n-1$. Furthermore, the central node has degree equal to $n-1$, whereas $d$ equals 1 for all other nodes in this network. In the ring network, $e$ equals $n$, and $d$ equals 2 for each node. 


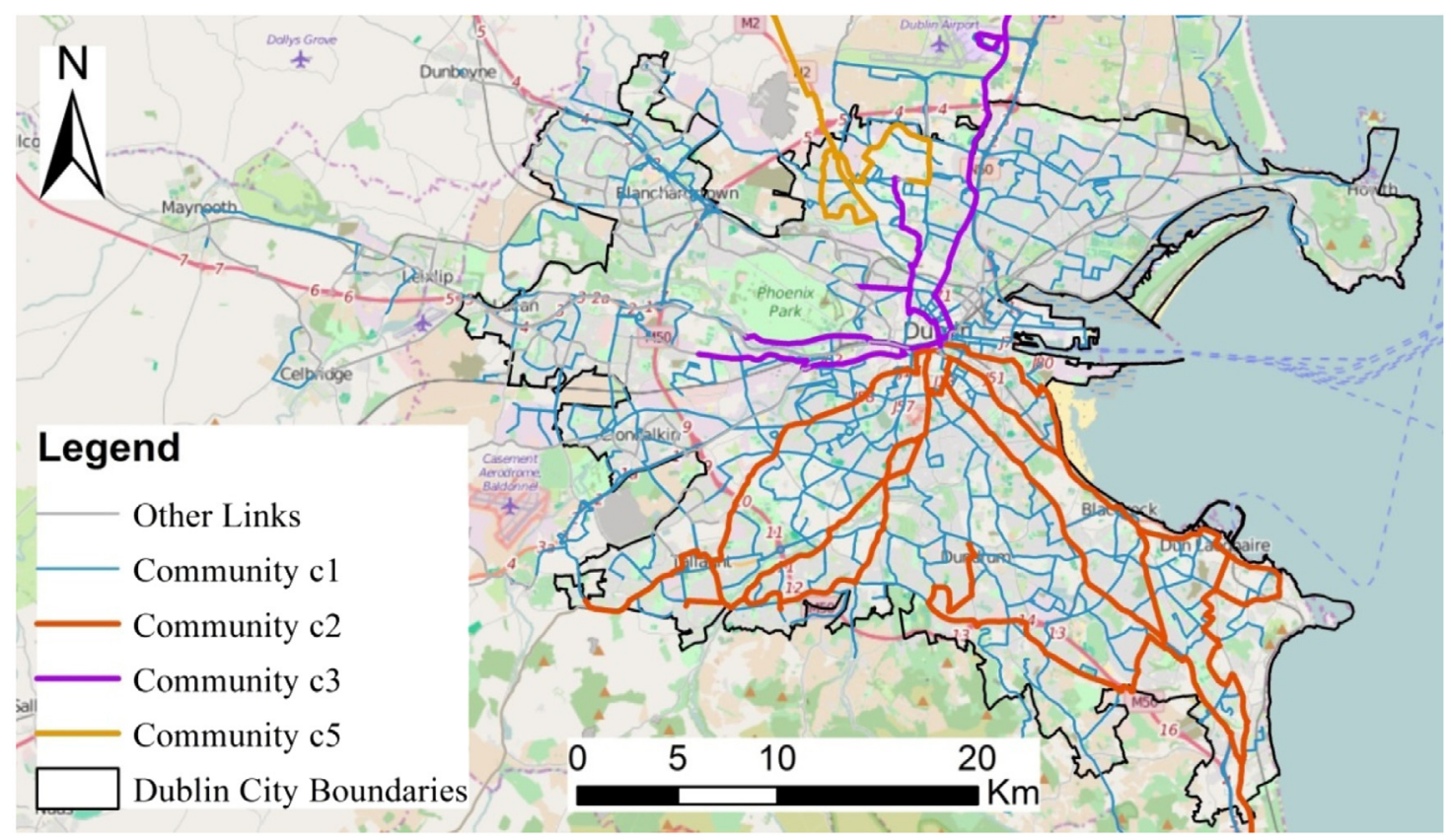

Fig. 2. Mapping the largest communities identified for the Dublin city using the bus network (Basemap: OpenStreetMap contributed by CC-BY-SA).

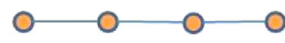

Line

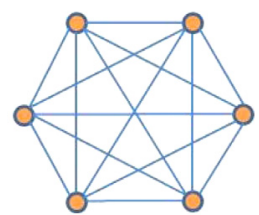

Mesh

(Fully Connected)<smiles>OC(O)(O)O</smiles>

Star

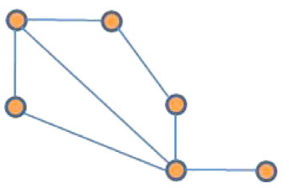

Mesh (partially Connected)

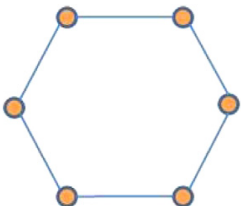

Ring

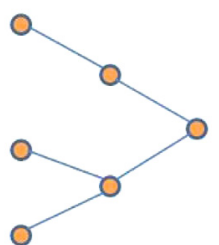

Tree

Fig. 3. Illustration of the basic network topologies.

In the fully connected mesh network in which all the nodes are interlinked, $e$ equals $n(n-1) / 2$, and $d$ equals $n-1$. In the partially connected mesh network, $e$ varies between $n$ and $n(n-1) / 2-1$, and $d$ varies between 1 and $n-1$. Finally, in the tree network (composed of an interconnected set of links with no circuits), e equals $n-1$, and $d$ ranges from 1 to $n-1$.

The illustration given above for the topologies can be directly applied to the bus network structure of the Dublin city. In the four communities, $c 1, c 2, c 3$, and $c 5$, the number of intra-community links (e) can exceed $n-1$ but must always be less than $n(n-1) / 2$. Since the maximum $d$ is greater than 3 for all the four communities, these communities can safely be assumed to be partially connected mesh networks.

\subsection{Analysis of the node influence}

The focus in this section continues to be placed on the five largest communities, $\{c 1, c 2, c 3, c 4, c 5\}$. We now examine the coefficients and other properties quantifying the node influence. 
Table 2

The total number of links and node degree in the basic types of network topologies.

\begin{tabular}{lll}
\hline Types of network topologies & Total number of links $(e)$ & Node degree $(d)$ \\
\hline Line & $n-1$ & 1 or 2 \\
Star & $n-1$ & 1 or $n-1$ \\
Ring & $n$ & 2 \\
Mesh (fully connected) & $n(n-1) / 2$ & $n-1$ \\
Mesh (partially connected) & {$[n, n(n-1) / 2)$} & {$[1, n-1]$} \\
Tree & $n-1$ & {$[1, n-1]$} \\
\hline
\end{tabular}

\subsubsection{Measure of roles of the nodes}

The first step of this analysis phase is to calculate the within-network degree $z$-score and the participation coefficient $p$-value for all nodes in the bus network, based on the formulas given in Eqs. (2) and (3). An illustration of this calculation is presented to provide more insight. Suppose that a node, $v_{i}$ belonging to the community $\mathrm{c} 1$ after the network partitioning, has a degree, $e_{v_{i}}$ equal to 44 , corresponding to its linkage with certain nodes of two communities, $\mathrm{c} 1$ and $\mathrm{c} 19$. Therefore, $e_{\mathrm{c} 1 v_{i}}$ (the number of links of node $v_{i}$ to the nodes in Community c1) equals 41 , and $e_{\mathrm{c} 19_{v_{i}}}$ (the number of links of node $v_{i}$ to the nodes in c19) equals 3 . Since this node, $v_{i}$ is only linked with nodes in $\mathrm{c} 1$ and $\mathrm{c} 19, e_{\mathrm{c} 1 v_{i}}$ and $e_{\mathrm{c} 19_{v_{i}}}$ have values greater than zero, while $e_{j_{v_{i}}}\{j=\mathrm{c} 2, \mathrm{c} 3, \ldots, \mathrm{c} 35\} \Lambda\{j \neq \mathrm{c} 19\}$ have zero values (note: the network is partitioned into 35 communities). The parameters $\bar{e}_{j_{v_{i}}}$ and $\sigma_{e_{j}}$ in Eq. (2) must be determined prior to the $z$-score calculation. The average of $e_{\mathrm{c} 1_{i}}$ over all the nodes of the community $\mathrm{c} 1$ is 6.7. The standard deviation of $e_{\mathrm{c} 1}$, over all the nodes of $\mathrm{c} 1$ equals 7.6. These values are derived respectively as follows:

$$
\begin{aligned}
& \bar{e}_{c 1_{i}}=\frac{1}{N_{\left\{v_{i} \in c 1\right\}}}\left(\sum_{i=1}^{N_{\left\{v_{i} \in c 1\right\}}} e_{c 1_{i}}\right)=\frac{1}{3474}\left(\sum_{i=1}^{3474} e_{\mathrm{c} 1_{i}}\right)=6.7 \\
& \sigma_{e_{j}}=\sqrt{\frac{1}{N_{\left\{v_{i} \in c 1\right\}}} \sum_{i=1}^{N_{\left\{v_{i} \in c 1\right\}}}\left(e_{\mathrm{c} 1_{i}}-\bar{e}_{\mathrm{c} 1_{i}}\right)^{2}}=\sqrt{\frac{1}{3474} \sum_{i=1}^{3474}\left(e_{\mathrm{c} 1_{i}}-6.7\right)^{2}}=7.6
\end{aligned}
$$

where $e_{\mathrm{c} 1}$ corresponds to the number of links of node $v_{i}$ to other nodes belonging to the community $\mathrm{c} 1$, and $N_{\left\{v_{i} \in c 1\right\}}$ corresponds to the number of nodes in this community. There are 3474 nodes in c1 (see Table 1). Accordingly, the $z$-score can be derived for node $v_{i}$ :

$$
z_{v_{i}}=\frac{e_{j_{v_{i}}}-\bar{e}_{j}}{\sigma_{e_{j}}}=\frac{41-6.7}{7.6}=4.5 .
$$

Correspondingly, the $p$-value for node $v_{i}$ is calculated as follows:

$$
\begin{aligned}
p_{v_{i}} & =1-\left[\left(\frac{e_{C 1_{v_{i}}}}{e_{v_{i}}}\right)^{2}+\left(\frac{e_{C 2 v_{v_{i}}}}{e_{v_{i}}}\right)^{2}+\cdots+\left(\frac{e_{\mathrm{C} 35_{v_{i}}}}{e_{v_{i}}}\right)^{2}\right] \\
& =1-\left[\left(\frac{e_{C 1_{v_{i}}}}{e_{v_{i}}}\right)^{2}+\left(\frac{e_{C 19_{v_{i}}}}{e_{v_{i}}}\right)^{2}\right]=1-\left[\left(\frac{41}{44}\right)^{2}+\left(\frac{3}{44}\right)^{2}\right]=0.13 .
\end{aligned}
$$

\subsubsection{Analysis of roles of the nodes}

Afterwards is the requirement to detect which nodes in the bus network are influential. A good strategy is to classify the nodes with $z$-score $\geq 2.5$ as module hubs, and nodes with $z$-score $<2.5$ as non-hubs [27]. The module hubs can be split further into sub-hubs on the basis of three different roles. "Provincial hubs", i.e., the hub nodes with the vast majority of links within their module ( $p$-value $\leq 0.3$ ), "connector hubs", i.e., hubs with many links to other modules within the bus network $(0.30<p$-value $\leq 0.75)$, and "kinless hubs", i.e., hubs with links homogeneously distributed among all modules ( $p$-value $>0.75$ ) [27]. Thus, the analysis of the current study first classifies the nodes into hubs and non-hubs using the threshold defined above for the $z$-score. However, since the vast majority of nodes in the bus network have a $p$-value of 0 , we make an adjustment to the threshold proposed for the $p$-value [27] to divide hub nodes more reasonably. The following rules are adopted for division of the hub nodes into two sub-hubs: "provincial hubs", i.e., hub nodes with all links within their module ( $p$-value $=0$ ), and "connector hubs", i.e., hubs with links to other modules ( $p$-value $>0$ ).

Consequently, approximately $4 \%$ of the nodes in the bus network are classified as hubs, and all the other nodes are classified as non-hubs. An inspection of the nodes identifies that less than $1 \%$ have a $p$-value greater than 0 , suggesting that very few nodes have links to other communities. Afterwards, we identify the most important nodes, including the 


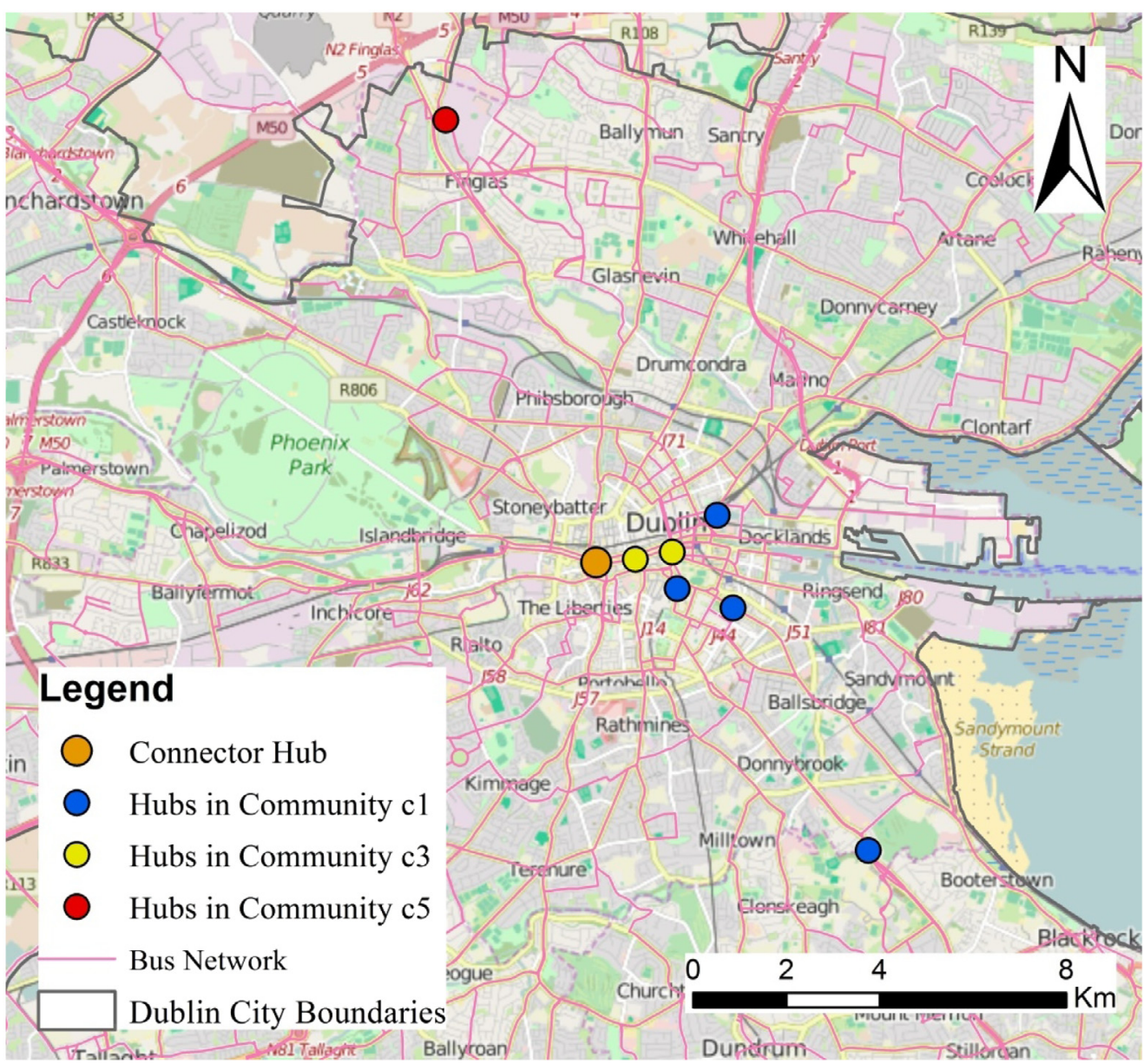

Fig. 4. Map of selected influential nodes in the bus network of Dublin city (Basemap: OpenStreetMap contributed by CC-BY-SA).

provincial hubs and connector hubs listed in Table 3. Specifically, in the four communities (i.e., c1, c2, c3, and c5), there are 150 provincial hubs and only one connector hub which is located in community c3.

We subsequently investigate the geographic characteristics of the nodes identified to be influential. A selection of these important nodes is shown in Fig. 4. It can be noted that only four hubs located within community c1 have a $z$-score $\geq 7.5$. These are the hubs in $\mathrm{c} 1$ presumed to have the highest amount of influence to the bus network. The hubs in the community c2 have not been mapped at all. While these hubs are assumed to play identical roles due to their similarity of $z$-score $\left(z_{v_{i}}=3.0\right)$ and play relatively small roles due to their relatively low $z$-score, the influence of these roles is relatively negligible. From the output of Fig. 4 it can be surmised that (1) the connector hub connecting the communities $c 3$ and $c 1$ is located in the center of the Dublin city, (2) three of the four primary provincial hubs ( $z$-score $\geq 7.5$ ) belonging to $c 1$ are located inside or near the city center, (3) the provincial hubs belonging to $\mathrm{c} 3$ are also located in the city center, and (4) in line with the expectation, the provincial hub in c5 is located near the community's centroid, which places its location in the Dublin Northside.

\subsection{Discussion}

This study has been undertaken to understand the mechanical statistics of the bus network structure in the Dublin city, Ireland's capital. Results of this empirical study have identified that the Dublin intraurban bus network is complex and characterized by a multi-community structure, and that its links are distributed heterogeneously between the bus transport nodes. Mapping the influential communities $(\mathrm{c} 2, \mathrm{c3}, \mathrm{c5})$ in the geographic space has revealed information intrinsically associated with the north-south socioeconomic subdivision of the city, further pointing out the theoretical meaningfulness of the partitioning strategy that was adopted for the analysis. With regard to the topological structure, this study identified the main communities as partially connected mesh networks. This indicates that the main communities are not likely to be regularly shaped and links are not relatively regularly distributed among nodes within them. Furthermore, several of the important nodes (bus network hubs) were located in the city center, which implies that intra-city bus stops are critical connectors of the entire bus network in Dublin. Assessment of the geographic location and shape of community c3 and its 
Table 3

Descriptive statistics of the influential nodes in the bus network of the Dublin city.

\begin{tabular}{|c|c|c|c|c|}
\hline Sub-type of role & $z$-score & $p$-value & Number of nodes & Community \\
\hline Provincial hub & $>2.5$ & 0 & $\begin{array}{r}118 \\
29 \\
2 \\
1\end{array}$ & $\begin{array}{l}\text { c1 } \\
\text { c2 } \\
\text { c3 } \\
\text { c5 }\end{array}$ \\
\hline Connector hub & 4.1 & 0.2 & 1 & c3 \\
\hline
\end{tabular}

provincial hubs revealed that the bus lines linking the northern with the western part of the city are likely to go through the city center. The location of the "connector hub" linking $c 3$ with $c 1$ additionally provides the information that the bus lines linking the northern part with the western part of the city are likely to be connected to other bus lines by a stop in the city center.

\section{Conclusion and future work}

Important insight has been gained in this study which contributes knowledge to the literature of assessing the urban network infrastructures. Specifically, the assessment of the community structure of the bus network in the current study, as well as the roles played critical network nodes can aid the undertaking of policy.

With regard to what remains unaddressed in the current study, future research is necessary to consider three essential aspects with reference to the intraurban bus networks. The first aspect concerns the fact that the spatial effects influencing the analysis of network structure are of increasing concern for network researchers. Modeling the spatial effects can provide useful insight into the future detection of communities. Secondly, other types of networks of similar structure (e.g., the Dublin metro network) can be analyzed for comparison purposes with the current study. Finally, given that bus and subway networks often interconnect for enhancing travel efficiency, it may be interesting to conduct analysis of the interaction between these two networks. Indeed, multiple networks also interconnect within cities (e.g., bus and rail with airway networks, bicycle networks). Thus an extension of the current study to the analysis of a hybrid network is necessary for uncovering additional useful network properties.

\section{Acknowledgments}

The corresponding author is thankful to the China Scholarship Council (CSC) for financial support with a Ph.D. scholarship. The authors acknowledge Dublinked for sharing research data used in this paper and two anonymous referees for their instructive comments.

\section{References}

[1] H. Soh, S. Lim, T. Zhang, et al., Weighted complex network analysis of travel routes on the Singapore public transportation system, Physica A 389 (24) (2010) 5852-5863.

[2] D.J. Watts, S.H. Strogatz, Collective dynamics of 'small-world' networks, Nature 393 (6684) (1998) 440-442.

[3] M. Marchiori, V. Latora, Harmony in the small world, Physica A 285 (3-4) (2000) 539-546.

[4] A. Réka, A.L. Barabási, Statistical mechanics of complex networks, Rev. Modern Phys. 74 (1) (2002) 47.

[5] V. Latora, M. Marchiori, Is the Boston subway a smallworld network? Physica A 314 (2002) 109-113.

[6] P. Sen, S. Dasgupta, A. Chatterjee, P.A. Sreeram, G. Mukherjee, S.S. Manna, Small-world properties of the Indian railway network, Phys. Rev. E 67 (2003) 036106.

[7] R. Guimerà, L.A.N. Amaral, Cartography of complex networks: Modules and universal roles, J. Stat. Mech. Theory Exp. (2005) P02001.

[8] J. Sienkiewicz, A. Janusz, Statistical analysis of 22 public transport networks in Poland, Phys. Rev. E 72 (2005) 46-50.

[9] Z. Xu, D.Z. Sui, Small-world characteristics on transportation networks: a perspective from network autocorrelation, J. Geogr. Syst. 9 (2) (2007) $189-205$.

[10] C. von Ferber, T. Holovatch, Y. Holovatch, V. Palchykov, Public transport networks: empirical analysis and modeling, Eur. Phys. J. B 68 (2) (2009) $261-275$.

[11] T. Jia, B. Jiang, Building and analyzing the US airport network based on en-route location information, Physica A 391 (15) (2012) $4031-4042$.

[12] Y. Li, W. Zhou, S.J. Guo, An analysis of complexity of public transportation network in Shanghai, Syst. Eng. 25 (2007) 38-41.

[13] M.E. Newman, D.J. Watts, Renormalization group analysis of the small-world network model, Phys. Lett. A 263 (4) (1999) 341-346.

[14] H. Zhang, P. Zhao, J. Gao, X. Yao, The analysis of the properties of bus network topology in Beijing basing on complex networks, Math. Probl. Eng. (2013) 694956.

15] S. Fortunato, Community detection in graphs, Phys. Rep. 486 (3) (2010) 75-174.

[16] M. Newman, M. Girvan, Finding and evaluating community structure in networks, Phys. Rev. E 69 (2004) 026113.

[17] R. Guimerà, M. Sales-Pardo, L.A.N. Amaral, Modularity from fluctuations in random graphs and complex networks, Phys. Rev. E 70 (2) (2004) 025101

18] M. Rosvall, C.T. Bergstrom, Maps of information flow reveal community structure in complex networks, Proc. Natl. Acad. Sci. 105 (4) (2008) 1118-1123.

19] A. Clauset, M.E.J. Newman, C. Moore, Finding community structure in very large networks, Phys. Rev. E 70 (6) (2004) 066111.

[20] J. Reichardt, S. Bornholdt, Statistical mechanics of community detection, Phys. Rev. E 74 (2006) 016110.

[21] P. Pons, M. Latapy, Computing communities in large networks using random walks, J. Graph Algorithms Appl. 10 (2) (2006) 191-218

[22] A.W. Rives, T. Galitski, Modular organization of cellular networks, Proc. Natl. Acad. Sci. 100 (2003) 1128-1133.

[23] J.D.J. Han, N. Bertin, T. Hao, D.S. Goldberg, G.F. Berriz, L.V. Zhang, D. Dupuy, A.J.M. Wallhout, M.E. Cusick, F.P. Roth, M. Vidal, Evidence for dynamically organized modularity in the yeast protein-protein interaction network, Nature 430 (6995) (2004) 88-93.

[24] G. Csárdi, T. Nepusz, The igraph software package for complex network research, Int. J. Complex Syst. 1695 (5) (2006) 1-9.

[25] B. Bicsi, Network Design Basics for Cabling Professionals, McGraw-Hill Professional, City, 2002.

[26] F. Xie, D. Levinson, Measuring the structure of road networks, Geogr. Anal. 39 (3) (2007) 336-356.

[27] R. Guimerà, S. Mossa, A. Turtschi, L.A.N. Amaral, The worldwide air transportation network: Anomalous centrality, community structure, and cities global roles, Proc. Natl. Acad. Sci. 102 (22) (2005) 7794-7799. 